\title{
How Should We Define Global Catastrophic BIOLOGICAL RisKs?
}

\author{
Dylan George
}

$\mathrm{T}$ He essay by the Johns Hopkins Center for Health Security on global catastrophic biological risks (GCBRs) presents an intriguing opportunity to assess the value of discriminating biologically derived risks from other global catastrophic risks (GCRs). Little research has focused on GCRs as a collective field of study, ${ }^{1}$ albeit individual types of GCRs have arguably received significant attention (eg, climate change, nuclear war). As such, their essay is thought-provoking and beneficial in refining our collective thinking about risks associated with biological events.

The authors present a definition of GCBR that emphasizes: (1) the "sudden" events with regard to the severity of impact, and (2) secondary impacts. First, they highlight a need to consider the speed with which an event occurs relative to our capacity to respond. In particular, certain acute, widespread biological events that exceed local capacities to effectively mitigate the event will be more concerning than those that either develop more slowly (eg, tuberculosis) or within our capacity to respond (eg, measles outbreak in California in 2014). The relatively short time frame in which the event unfolds is an important and valuable contribution in this definition.

As a contrast, early definitions of "pandemic" focused fundamentally on transmissibility or, rather, how an infectious disease spreads internationally. ${ }^{2-4}$ Definitions were further clarified after the $2009 \mathrm{H} 1 \mathrm{~N} 1$ influenza pandemic to include additional emphasis on clinical severity and the potential for significant morbidity and mortality, ${ }^{5,6}$ but extent of transmission remained the dominant factor. This earlier concept of a pandemic was insufficient because it did not explicitly consider the speed of transmission and thereby conflates chronic pandemics (eg, HIV/AIDS, tuberculosis) and acute pandemics (eg, influenza, dengue, SARS). A valuable differentiator of risk has not been sufficiently emphasized since the response needs and severity of impact of a chronic pandemic will likely be much different from those of an acute pandemic. As such, the Center's definition of GCBRs provides a useful contribution, because it constrains thinking to "sudden" biological events that could outstrip currently resourced local capacities for mitigation.

Further, their definition considers secondary impacts of the biological event by explicitly emphasizing how certain biological events can destabilize national and international security. Specifically, their definition of GCBR considers the impact on "national governments, international relationships, economies, societal stability, or global security" in addition to mass mortality events. This is a reasonable and welcome approach, but it is not clear that these impacts would be exclusive to biological events. Other GCRs undoubtedly would cause secondary impacts as well. For example, the impact of a nuclear detonation in a civilian population would clearly have immediate mass mortality effects, but it would also have the secondary impacts on all components considered for GCBRs (ie, national governments, international relationships, economies, societal stability, or global security). As such, it is unclear that the definition of GCBR sufficiently discriminates the uniqueness of biologically derived catastrophic risks from other GCRs by including secondary impacts as a defining concept.

An additional concept should be considered to further discriminate GCBRs from other GCRs. While the authors insightfully emphasize the secondary impacts, further thought should be given to how GCBRs uniquely disrupt social connections in ways that other GCRs do not. The majority of GCBRs, but not all, will be infectious diseases, as the authors point out. The social erosion generated by a contagious

(C) Dylan George, 2017; Published by Mary Ann Liebert, Inc. This Open Access article is distributed under the terms of the Creative Commons Attribution Noncommercial License (http://creativecommons.org/licenses/by-nc/4.0/) which permits any noncommercial use, distribution, and reproduction in any medium, provided the original author(s) and the source are credited. 
biological event could be unique from other GCRs. For example, most GCBRs will likely be transmitted among humans via respiratory routes or require relatively close contact for transmission (eg, influenza). For human transmitted pathogens, contagiousness could engender significant distrust among individuals, communities, or nations that would exacerbate the secondary impacts on societal stability. Examples of this sort of distrust, at smaller scales, includes the attempts at closing international flights during the Ebola outbreak in West Africa, ${ }^{7}$ inappropriately quarantining responders when they returned to the United States, ${ }^{8}$ and the intense social stigma associated with HIV/AIDS. ${ }^{9}$ This social stress may be less intense with environmentally transmitted or vector-borne pathogens, given transmission will not be as overtly driven by other humans. If so, then the axis of interest would not necessarily be biological but would be the potential sociological implications of a GCBR. Biological events of sufficient size could disrupt societal stability in unique ways not seen among other GCRs.

While the essay does not fully address why there is a need to partition out GCBRs as a subcategory of GCRs, it does stimulate useful thinking about biological GCRs. So, the authors have accomplished their objective in writing their essay: encouraging collective thinking about global catastrophic biological risks.

\section{REFERENCES}

1. Bostrom N. The end of humanity. TedXOxford. 2013. https:// www.youtube.com/watch?v=P0Nf3TcMiHo. Accessed June 21, 2017.
2. Doshi P. The elusive definition of pandemic influenza. Bull World Health Organ 2011;89:532-538.

3. Kelly $\mathrm{H}$. The classical definition of a pandemic is not elusive. Bull World Health Organ 2011;89:540-541.

4. Cohen E. 2009. When a pandemic isn't a pandemic. CNN.com. May 4, 2009. http://www.cnn.com/2009/HEALTH/05/04/swine. flu.pandemic/index.html?iref=nextin. Accessed June 21, 2017.

5. Reed C, Biggerstaff M, Finelli L, et al. Novel framework for assessing epidemiologic effects of influenza epidemics and pandemics. Emerg Infect Dis 2013;19(1):85-91.

6. McNeil DG Jr. W.H.O. to rewrite its pandemic rules. New York Times May 22, 2009. http://www.nytimes.com/2009/ 05/23/health/policy/23who.html. Accessed June 21, 2017.

7. Ebola: airlines cancel more flights to affected countries. The Guardian August 22, 2014. https://www.theguardian.com/ society/2014/aug/22/ebola-airlines-cancel-flights-guinea-liberiasierra-leone. Accessed June 21, 2017.

8. Johnson A. Kaci Hickox, Maine nurse quarantined in Ebola scare, sues New Jersey Gov. Chris Christie. NBC News October 22, 2015. http://www.nbcnews.com/storyline/ebola-virus-out break/kaci-hickox-maine-nurse-quarantined-ebola-scare-sues-newjersey-n449491. Accessed June 21, 2017.

9. Parker R, Aggleton P. HIV and AIDS-related stigma and discrimination: a conceptual framework and implications for action. Soc Sci Med 2003;57(1):13-24.

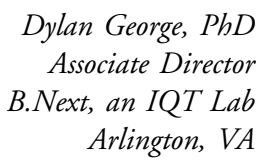

Email: dgeorge@iqt.org 